'Facultad de Medicina Clínica Alemana Universidad del Desarrollo. Santiago, Chile. aTecnólogo Médico.

${ }^{\text {b} M a g i ́ s t e r ~ e n ~ E d u c a c i o ́ n . ~}$ 'Bioquímico.

dProfesor de Enseñanza Media. ePhD

Recibido el 30 de mayo de 2017, aceptado el 3 de octubre de 2017.

Correspondencia a: Paulette Conget

Facultad de Medicina Clínica Alemana-Universidad del Desarrollo.

Av. Las Condes 12.438. Santiago, Chile. pconget@udd.cl

\section{La creencia epistemológica constructivista sobre el conocimiento científico varía en función del año de formación en los estudiantes de Medicina pero no en los estudiantes de otras carreras de la salud}

\author{
XIMENA LAZCANO ${ }^{1, \mathrm{a}, \mathrm{b}}$, FRANCISCO VILLALÓN $^{1}$, \\ SOLEDAD VERA ${ }^{1, \mathrm{a}}$, PAULETTE CONGET ${ }^{1, \mathrm{c}, \mathrm{d}, \mathrm{e}}$
}

\section{The constructivist epistemological belief about scientific knowledge varies according to the year of training in medical students but not in students of other health careers}

\begin{abstract}
Background: To optimize the teaching-learning process it is fundamental to know the representations that students have regarding knowledge. Epistemological beliefs are implicit theories that guide the practical actions of people. Aim: To characterize and compare epistemological beliefs regarding the nature and acquisition of scientific knowledge of health career students. Material and Methods: Between 2012 and 2013, 726 students coursing first, third or fifth year from six health careers answered a validated questionnaire that includes closed and open questions aimed to characterize their epistemological beliefs about scientific knowledge. Results: Irrespective of the career, when students had to select predefined answers, most of them appeared as constructivists (61\%). On the other hand, when they had to argue, the majority seemed objectivist (47\%). First-year medical students have the highest frequency of constructivist epistemological beliefs (56\%). Paradoxically, the lowest percentage is found (34\%) in the fifth year. The students of the health careers, in particular those of Medicine, recognize that knowledge is not acquired immediately (83\%) and that its distribution is shared (92\%). Conclusions: Discordance between selections and arguments suggests that epistemological sophistication is achieved declaratively but not practically. The lower proportion of students who presented constructivist beliefs in the fifth year compared to first year of Medicine could be associated with the pedagogical approaches used in the different cycles of the career.
\end{abstract}

(Rev Med Chile 2017; 145: 1184-1192)

Key words: Health Education; Knowledge; Students, Medical.
L a epistemología, que tiene su génesis en la filosofía, se ocupa del origen, naturaleza, límites, métodos y justificación del conocimiento humano. Las creencias epistemológicas son teorías implícitas que orientan la acción prác- tica de las personas ${ }^{1,2}$. Ellas transitan desde miradas simples hasta visiones reflexivas ${ }^{3-7}$. La concepción del conocimiento como algo relativamente inmodificable, que se adquiere rápidamente y que reside en la autoridad devela una epistemología 
ingenua. Por el contrario, cuando el conocimiento aparece como algo incierto, complejo y que puede ser aprendido gradualmente la creencia epistemológica se califica como sofisticada. Así, una persona que se reconoce receptiva, se adapta al objeto y declara que el conocimiento es algo absoluto presenta una epistemología objetivista. Si el sujeto percibe el conocimiento como algo no absoluto y que depende del punto de vista del emisor de la información se considera relativista. Una individuo que concibe el conocimiento como un proceso continuo, complejo y evolutivo presenta una epistemología constructivista. Cuando ninguna de las tres categorías anteriores está claramente marcada la creencia de la persona es mixta ${ }^{8}$.

En particular respecto del conocimiento científico se han propuesto diversas sistematizaciones para las creencias epistemológicas. Schommer-Atkins las organiza en un modelo multidimensional cuyos componentes son independientes y no necesariamente secuenciales ${ }^{9}$. Así, agrupa las creencias epistemológicas respecto del conocimiento científico en las dimensiones naturaleza y adquisición. Dentro de la naturaleza del conocimiento científico se incluyen los componentes certeza y criterio de verdad. El primero dicotomiza al conocimiento como algo absoluto o como algo provisional. El segundo considera las fuentes de evidencia, los modelos de justificación, la simplicidad o complejidad de los recursos y el carácter absoluto o problemático de las justificaciones. Por su parte, la dimensión adquisición presenta los componentes inmediatez y distribución. El primero se refiere a la celeridad para adquirir el conocimiento científico pudiendo ser instantánea o progresiva. El segundo supone que quienes adquieren el conocimiento son expertos o personas inteligentes (conocimiento restringido) o bien que éste está uniformemente distribuido (conocimiento compartido). En otros modelos, este último componente se denomina fuente del conocimiento y se tipifica en función de la autoridad y/o inteligencia ${ }^{10}$. Basado es estas dimensiones y componentes se han desarrollado y validado cuestionarios estandarizados que permite conocer las creencias, preferencias, justificaciones y posiciones epistemológicas respecto del conocimiento científico de las personas ${ }^{8,11-13}$.

A la fecha, se han explorado las teorías implícitas sobre el conocimiento de escolares, estudiantes de pregrado y posgrado de distintas áreas del conocimiento ${ }^{2,14-16}$. Se ha mostrado que cuando el estudiante considera el conocimiento como algo simple, manejable, cierto y dependiente de la autoridad; simplifica la información, formula conclusiones poco elaboradas y escoge respuestas simples ${ }^{17}$. Diversos autores han analizado la relación entre creencias epistemológicas y comprensión lectora ${ }^{18,19}$. También, entre creencias epistemológicas y rendimiento académico ${ }^{10,20-22}$. Es así que cuanto más sofisticadas son éstas, los estudiantes tienen mejores resultados académi$\cos ^{12}$. Algunos autores concluyen que las creencias y aproximaciones al aprendizaje progresan a medida que los estudiantes avanzan en su proceso formativo ${ }^{14-16,23}$. Otros, no encuentran relaciones estadísticamente significativas. Un tercer grupo, encuentra una relación inversa.

En la formación médica se han estudiado las creencias epistemológicas respecto del conocimiento científico de cohortes de estudiantes y profesores. En los primeros se han descrito relaciones inversas entre edad y maduración de las dimensiones, y entre inmadurez y notas ${ }^{16,24}$.

Hasta donde nosotros conocemos, no hay estudios en que se comparen las creencias epistemológicas respecto del conocimiento científico entre estudiantes de distintas carreras de la salud. Así, el objetivo de este trabajo fue caracterizar y comparar las creencias epistemológicas respecto de la naturaleza y adquisición del conocimiento científico de estudiantes de carreras de la salud impartidas en Chile. Para ello, se enrolaron 726 estudiantes de primer, tercer o quinto año de seis carreras de la salud de la Facultad de Medicina Clínica Alemana-Universidad del Desarrollo quienes respondieron un cuestionario validado que incluye preguntas cerradas y abiertas dirigidas a caracterizar sus creencias epistemológicas respecto del conocimiento científico. Los datos recabados se analizaron en función de los componentes certeza, criterio de verdad, inmediatez y distribución. También, de las preferencias, justificaciones y posiciones epistemológicas. Todo lo anterior para la muestra total y en función de las variables sexo, carrera y año de la carrera cursado.

\section{Material y Método}

\section{Población de estudio}

Setecientos veinte y seis estudiantes de primer, tercer o quinto año de las carreras de Medicina, 
Odontología, Kinesiología, Fonoaudiología, Enfermería y Tecnología Médica de la Facultad de Medicina Clínica Alemana-Universidad del Desarrollo de Santiago de Chile aceptaron voluntariamente participar en el estudio.

El protocolo fue aprobado por el Comité de Ética de la Investigación de la Facultad de Medicina Clínica Alemana-Universidad del Desarrollo.

\section{Cuestionario}

Se aplicó un cuestionario que permite distinguir las creencias epistemológicas respecto del conocimiento científico acorde a las dimensiones propuestas por Schommer-Atkins, previamente estandarizado y validado en Chile ${ }^{8,9}$ (Figura Supl. 1). Este instrumento contiene 22 preguntas cerradas que se responden utilizando una escala tipo Likert tal que "desacuerdo total" se valora como 1 y "total acuerdo" como 6. También, incluye una pregunta en que el sujeto debe escoger una de seis afirmaciones (pregunta A) y debe justificar su elección (pregunta B). Además, una pregunta que refiere a una situación dada y en la que el sujeto debe tomar una postura (pregunta C) y fundamentarla (pregunta D). Las preguntas 1 a 6 configuran el componente certeza de la dimensión naturaleza del conocimiento. Las preguntas 7 a 22 configuran los componentes inmediatez y distribución de la dimensión adquisición del conocimiento. Las preguntas A, B y C dan cuenta de la preferencia, la justificación y la posición epistemológica del encuestado, respectivamente. La pregunta D revela su criterio de verdad.

\section{Recogida y almacenamiento de datos}

Los datos fueron recogidos en el período 20122013. Los estudiantes respondieron el cuestionario al inicio de una clase y dispusieron de $10 \mathrm{~min}$ para ello. Los datos recabados fueron almacenados en una base de datos creada en el programa Excel.

\section{Análisis de datos}

Para el componente certeza de la dimensión naturaleza del conocimiento las categorías objetivista, relativista, constructivista o mixto se asignaron en función del valor de la media obtenida en las preguntas 1 a 6 . El criterio de verdad y la justificación epistemológica se infirieron a partir de las respuestas a las preguntas $\mathrm{D}$ y $\mathrm{B}$, respectivamente. El análisis de dichas respuestas fue hecho por tres jueces independientes que utilizaron una misma pauta con criterios pre-establecidos ${ }^{8}$. El coeficiente Kappa ponderado para más de dos jueces para criterio de verdad fue 0,87 y para la justificación epistemológica fue 0,82 . La posición epistemológica se configuró a partir de la repetición tres o más veces de una misma categoría en certeza, preferencia epistemológica, justificación epistemológicas y criterio de verdad. Si se presentaba inconsistencia, el sujeto fue categorizado como mixto.

Las categorías inmediata o no inmediata y restringido o compartido de la dimensión adquisición del conocimiento se asignaron en función del valor de la media obtenida en las preguntas 7 a $22^{8}$.

La confiabilidad del instrumento se calculó utilizando el coeficiente alpha de Cronbach, obteniéndose una correlación general de 0,76.

\section{Análisis estadístico}

Para cada una de las variables estudiada se realizó análisis de proporción. Se utilizó $\chi^{2}$ para la comparación de los porcentajes obtenidos, con posterior análisis de residuos estandarizados (RE). Todo lo anterior se realizó en el paquete estadístico SPSS versión 15.0. Se consideraron como estadísticamente significativos valores de $\mathrm{p}<0,05$.

\section{Resultados}

Los 726 estudiantes que participaron voluntariamente en el estudio se distribuyeron en proporciones similares entre las seis carreras de la salud incluidas en el estudio (Tabla $1 \mathrm{~A}$ ).

La distribución de sexo en la muestra fue $71 \%$ mujeres y $29 \%$ hombres, encontrándose la mayor proporción de mujeres en Enfermería $(\mathrm{RE} 3,4)$ y Fonoaudiología (RE 2,1) y la menor, en Medicina $(\mathrm{RE}-2,4)(\mathrm{gl} 5, \mathrm{p}=0,000)($ Tabla $1 \mathrm{~B})$.

Como se observa en la Figura 1, tanto para certeza como para preferencia y justificación epistemológicas predomina en la muestra estudiada la creencia constructivista $(64,61$ y $62 \%$, respectivamente) por sobre la objetivista (19, 18 y $21 \%$, respectivamente) y la relativista (17, 21 y $17 \%$, respectivamente) (gl 2, p = 0,000). En cambio, en posición epistemológica aparecieron muchos estudiantes categorizados como mixtos dada la inconsistencia de sus respuestas (29\%). En criterio de verdad la proporción predominante fue 
Tabla 1. Sujetos de estudio

A. Distribución por carrera y sexo

\begin{tabular}{|c|c|c|c|c|}
\hline & $\begin{array}{c}\text { I año } \\
\text { (número) }\end{array}$ & $\begin{array}{c}\text { III año } \\
\text { (número) }\end{array}$ & $\begin{array}{c}\text { V año } \\
\text { (número) }\end{array}$ & $\begin{array}{c}\text { Total } \\
\text { (número) }\end{array}$ \\
\hline Medicina & 59 & 48 & 32 & 139 \\
\hline Odontología & 51 & 53 & 19 & 123 \\
\hline Kinesiología & 53 & 48 & 40 & 141 \\
\hline Fonoaudiología & 46 & 24 & 14 & 84 \\
\hline Enfermería & 56 & 50 & 21 & 127 \\
\hline Tecnología médica & 64 & 35 & 13 & 112 \\
\hline Total & 329 & 258 & 139 & 726 \\
\hline
\end{tabular}

B. Distribución por carrera y año cursado

\begin{tabular}{|lcc|}
\hline & $\begin{array}{c}\text { Mujeres } \\
\text { (\%) }\end{array}$ & $\begin{array}{c}\text { Hombres } \\
\text { (\%) }\end{array}$ \\
\hline Medicina & $55^{*}$ & 45 \\
\hline Odontología & 59 & 41 \\
\hline Kinesiología & 64 & 36 \\
\hline Fonoaudiología & $90^{*}$ & 10 \\
\hline Enfermería & $96^{*}$ & 4 \\
\hline Tecnología médica & 69 & 31 \\
\hline Total & 71 & 29 \\
\hline
\end{tabular}
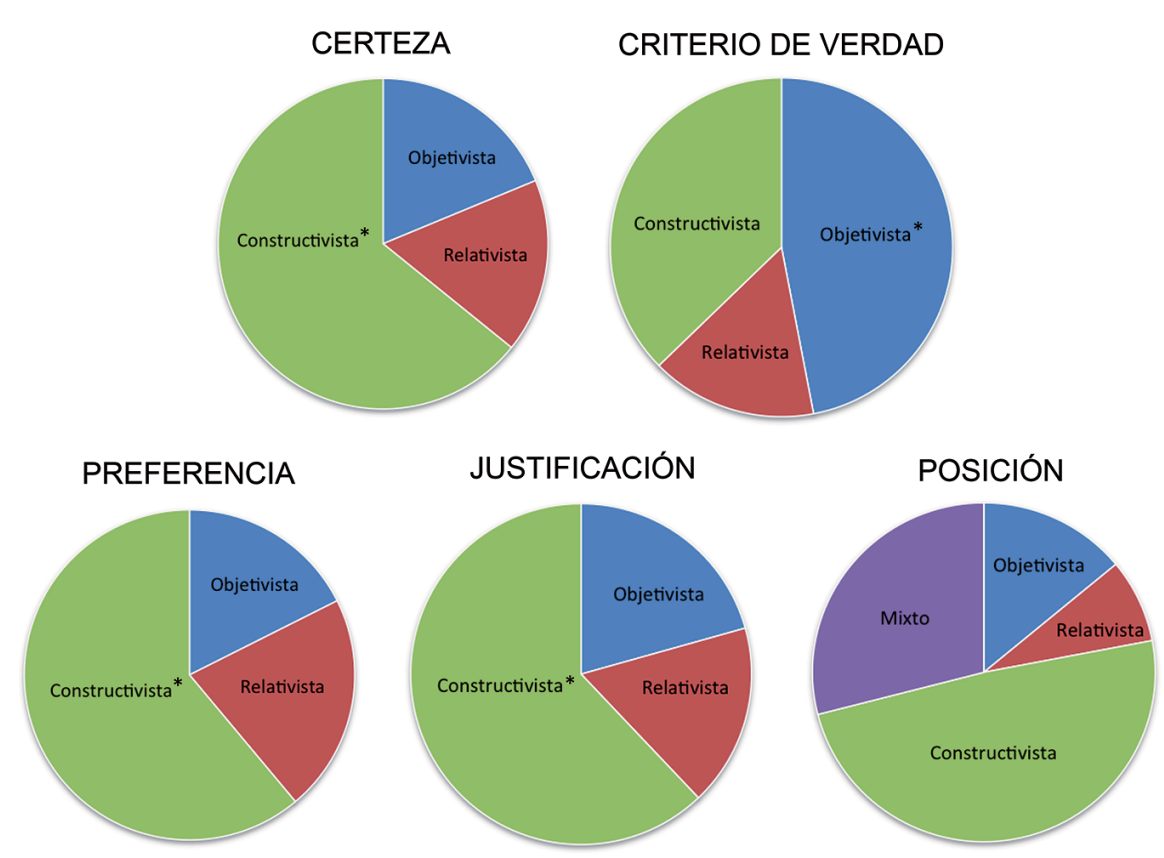

Figura 1. Distribución de creencias epistemológicas sobre la naturaleza del conocimiento científico de estudiantes de carreras de la salud. 
la objetivistas $(47 \%)(\mathrm{gl} 2, \mathrm{p}=0,000)$. El análisis de los datos obtenidos para la muestra total no reveló diferencias en la creencias epistemológicas respecto del conocimiento científico entre mujeres y hombres (datos no mostrados).

Al comparar entre carreras de la salud no se encontraron diferencias respecto de certeza, criterio de verdad, preferencia o posición pero si en justificación epistemológica ( $\mathrm{gl} 10, \mathrm{p}=0,004$ ) (Figura 2). Destacó una mayor proporción de objetivistas en Odontología (RE 2,2) y de relativistas en Tecnología Médica (RE 2,6). También, una baja proporción de relativistas en Enfermería (RE -2,2). Estas diferencias se mantuvieron luego del control de variables confundentes, particularmente sexo (gl 10, $\mathrm{p}=0,005)$.

Al desagregar la muestra por año de la carrera cursado se observaron diferencias en certeza ( $\mathrm{gl}$ $10, \mathrm{p}=0,009)$, criterio de verdad $(\mathrm{gl} \mathrm{10}, \mathrm{p}=0,006)$

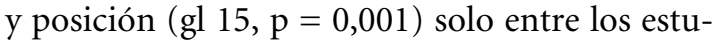
diantes de primer año. Siendo los estudiantes de primer año de Medicina quienes presentaron la frecuencia más alta de creencias epistemológicas constructivistas (certeza: 78\%, RE 1,3; criterio de verdad: $56 \%$, RE 2,1 y posición: $71 \%$, RE 1,9 ).

$\mathrm{Al}$ analizar por carrera, en Medicina se observa un cambio significativo en las creencias epistemológicas sobre la certeza del conocimiento entre distintos años de la carrera $(\mathrm{gl} 8, \mathrm{p}=0,012)$. El porcentaje de estudiantes constructivista disminuye desde $78 \%$ en primer año (RE 1,2 ) a $67 \%$ en tercer año (RE 0,1$)$ para llegar a $41 \%$ en quinto año (RE -1,7) (Figura 3). Lo mismo se observa para criterio de verdad ( $\mathrm{gl} 4, \mathrm{p}=0,04)$ y posición epistemológica ( $\mathrm{gl} \mathrm{6,p}=0,042)$ tal que en primer año los constructivistas corresponden a $56 \%(\mathrm{RE}$ 1,6) y $71 \%$ (RE 1,8), en tercer año a $31 \%(\mathrm{RE}-1,2)$ y $43 \%(\mathrm{RE}-1)$ y en quinto año a $34 \%(\mathrm{RE}-0,7)$ y $37 \%$ (RE - 1,3).

La mayoría de los estudiantes reconoce que la adquisición del conocimiento no es inmediata $(83 \%, p=0,000)$. Aparecen diferencias entre carreras ( $\mathrm{gl} \mathrm{5,p}=0,000$ ), encontrándose una mayor proporción de creencias de inmediatez en

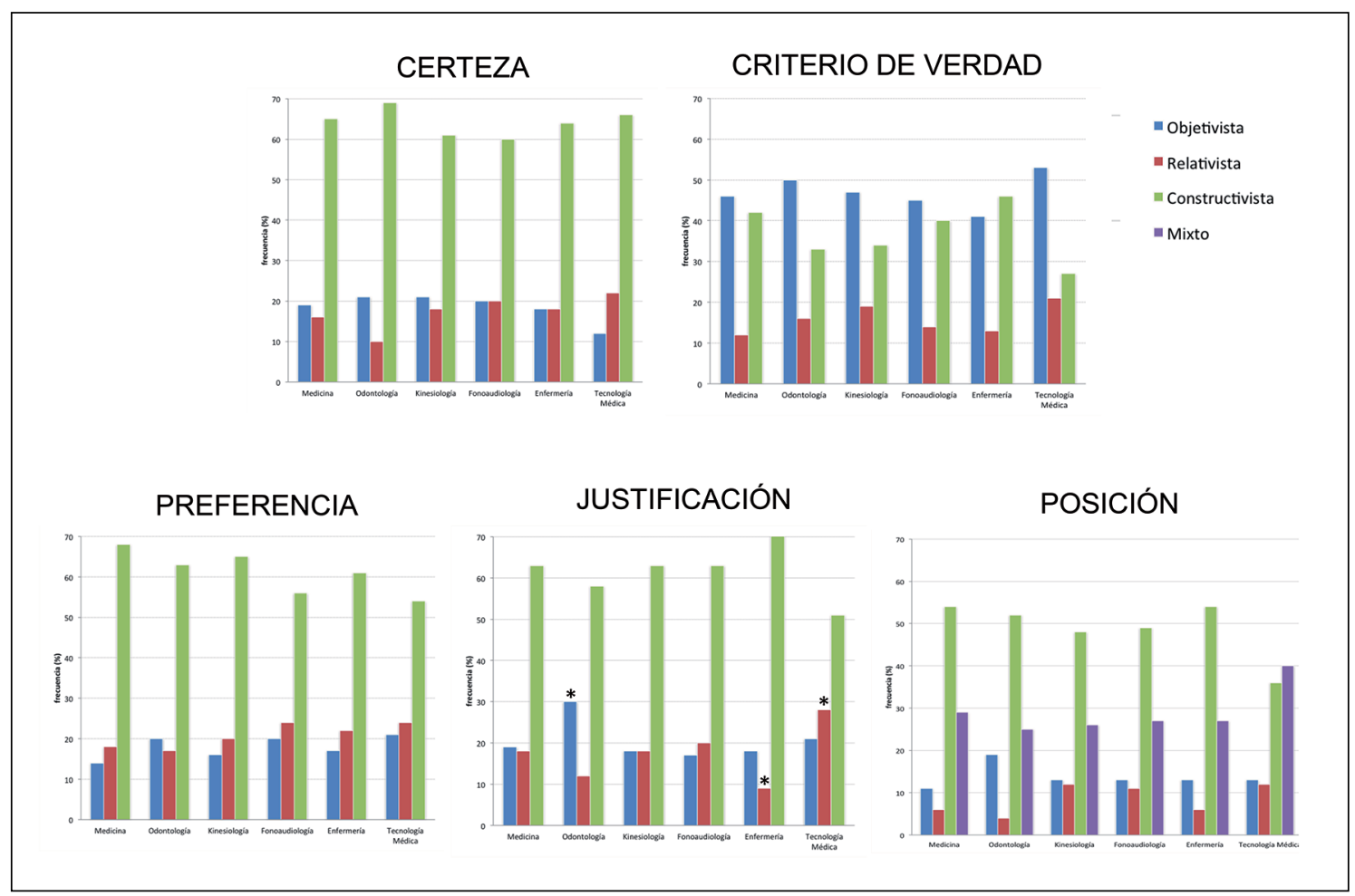

Figura 2. Distribución de creencias epistemológicas sobre la naturaleza del conocimiento científico de estudiantes de carreras de la salud en función de la carrera estudiada. 


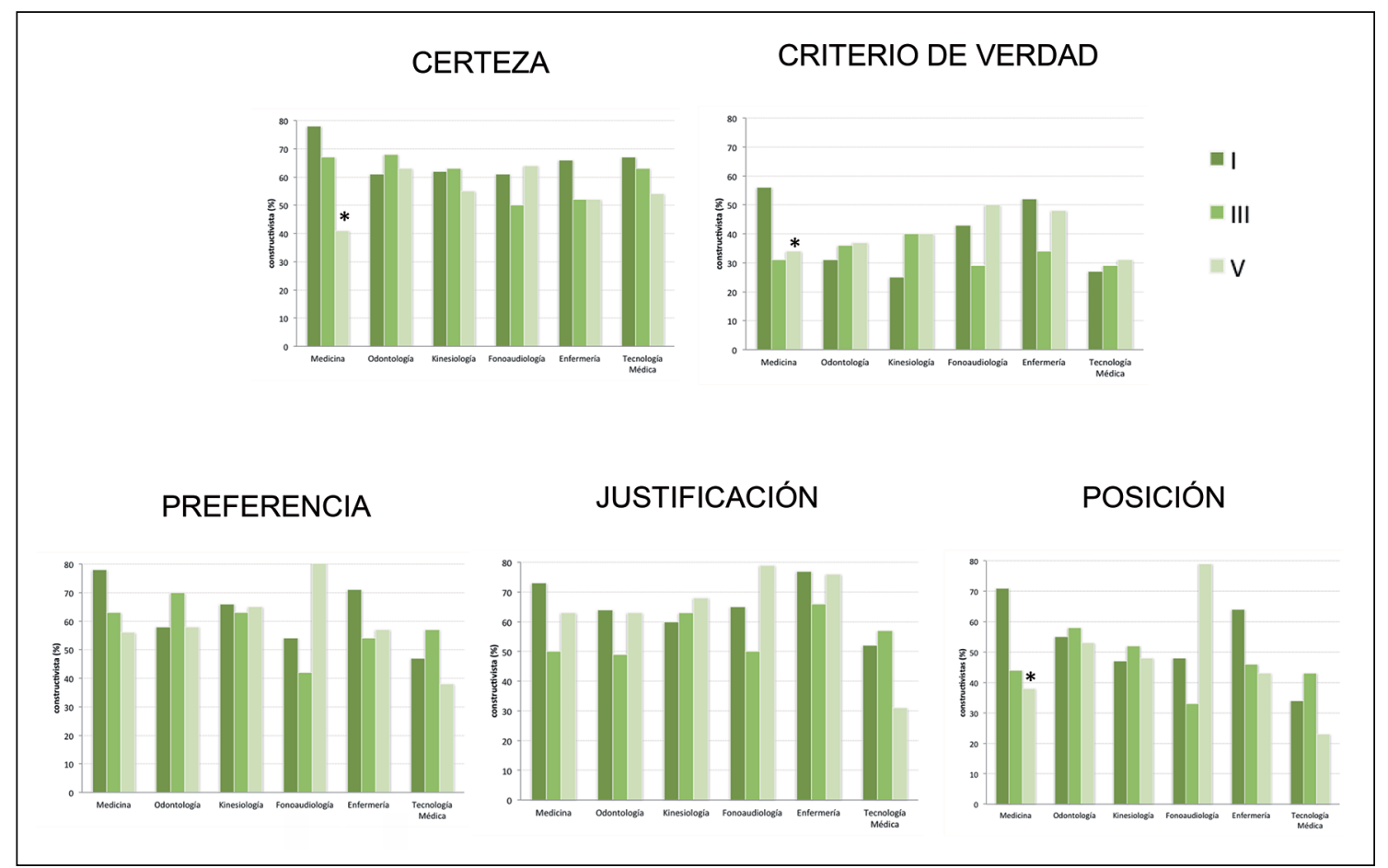

Figura 3. Frecuencia de estudiantes de carreras de la salud que presenta creencia epistemológica constructivista sobre la naturaleza del conocimiento científico en función del año cursado.

la adquisición del conocimiento en Enfermería (RE 2,4) y Tecnología Médica (RE 2,1), y siendo la menor en Medicina (RE -3,6) (Figura 4).

La casi totalidad de los estudiantes de las carre- ras de la salud cree que la distribución del conocimiento es compartida $(92 \%, \mathrm{p}=0,000)$. No se observan diferencias significativas al respecto entre sexos, carreras o año de carrera cursado (Figura 4).

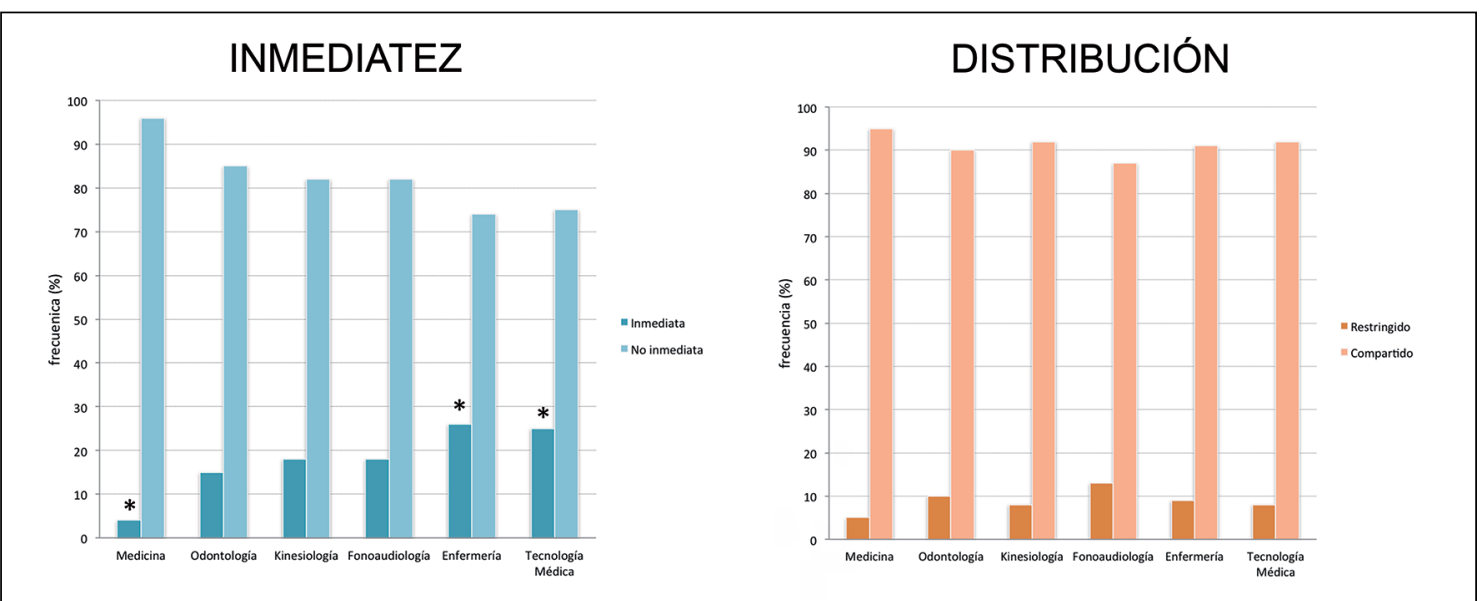

Figura 4. Distribución de creencias epistemológicas sobre la adquisición del conocimiento científico de los estudiantes de carreras de la salud en función de la carrera cursada. 


\section{Discusión}

Caracterizar las creencias epistemológicas de los estudiantes puede ser útil para predecir el rendimiento pero más importante aun, para orientar las decisiones pedagógicas ${ }^{25}$. En este estudio se presentan las creencias epistemológicas respecto del conocimiento científico de estudiantes de seis carreras de la salud de la Facultad de Medicina Clínica Alemana-Universidad del Desarrollo. Los jóvenes enrolados en el estudio comparten la distribución de sexo y edad, y los puntajes de selección universitaria de los estudiantes de carreras de la salud impartidas en otras universidades chilenas.

En la muestra total se encontró una discordancia entre las respuestas de elección versus aquellas de argumentación. Este hallazgo coincide con lo descrito por otros autores ${ }^{8}$. Y se explica puesto que responder una pregunta cerrada implica seleccionar una respuesta ofertada y no desarrollar una propia. Además, es una tarea simple y sesgada hacia lo socialmente correcto. En cambio, al argumentar la persona expone libremente sus teorías implícitas. Todo lo anterior confluye para que en muchas personas la sofisticación epistemológica se alcance en lo declarativo pero no en la práctica.

En la presente investigación no se encontraron diferencias entre mujeres y hombres respecto de la naturaleza y la adquisición del conocimiento. Este resultado era esperado puesto que en ningún estudio reportado a la fecha las creencias epistemológicas respecto del conocimiento científico dependen del sexo de la persona $a^{5,26-28}$.

$\mathrm{Al}$ examinar la variable carrera no se hallaron diferencias estadísticamente significativas. Sin perjuicio de lo anterior, la frecuencia más alta de estudiantes constructivistas se encontró en primer año de la carrera de Medicina. Además, ellos son los que mayoritariamente consideran que el conocimiento no se adquiere en forma inmediata. Ambos parámetros indican una madurez epistemológica, la cual se relaciona con alto rendimiento escolar y mayores puntajes de selección universitaria. Así nuestros datos coinciden con aquellos estudios que muestran una relación directa entre mayor sofisticación en las creencias epistemológicas y mejor rendimiento académico $3,18,20,29-31$.

$\mathrm{Al}$ analizar la variable año de la carrera cursado solo se encontraron diferencias estadísticamente significativas para la carrera de Medicina.
Inesperadamente, la frecuencia de estudiantes constructivistas para la dimensión naturaleza (certeza, criterio de verdad y posición) fue menor en quinto año que en los años precedentes. Esto refleja una regresión en la sofisticación epistemológica. Desarmonías en el desarrollo de las creencias epistemológicas, tal que evolucionan de manera independiente han sido previamente des$\operatorname{critas}^{1,2,9,32}$. Coincidentemente, para estudiantes de carreras de Pedagogía impartidas en Chile no solo se observó una involución de las creencias epistemológicas entre primer y cuarto año, si no aun más se evidenció un retroceso en éstas después de haber cursado una asignatura sobre aprendizaje $\mathrm{e}^{33}$.

A falta de bibliografía que aporte explicaciones para el regresión observada en los estudiantes de Medicina, hipotetizamos que ella está condicionada por los enfoques pedagógicos utilizados en los distintos ciclos de la carrera de Medicina. Mientras en los primeros años de la carrera (ciclo básico) se privilegian la reflexión y el análisis critico en escenarios simulados (casos clínicos, presentaciones clínicas, práctica con fantomas, pacientes estandarizados, proyectos de investigación), a partir del tercer año (ciclo pre-clínico) y consolidándose en quinto año (ciclo clínico) la actividad fundamental que realizan los estudiantes es la práctica clínica supervisada en ocasiones bastante protocolizada. Así, en primer año el estudiante es inducido a explorar la mayor cantidad posible de explicaciones y puede equivocarse sin que ello tenga repercusiones en un otro; en quinto año, sus reflexiones deben ser rápidas y traducirse en decisiones concretas y correctas. Esto último, por economía cognitiva, inseguridad y pragmatismo podría automatizarse a expensas de habilidades cognitivas de baja complejidad, llevando a un retroceso desde las creencias constructivistas hacia las objetivistas.

Otras variables que puedan dar cuenta de los resultados obtenidos son aspectos disciplinarios, actitudinales, motivacionales y los modelos profesionales a que están expuestos los estudiantes.

Puesto que la evolución de las creencias epistemológicas entre los niveles iniciales y terminales de formación debiera ser desde concepciones ingenuas a sofisticadas ${ }^{20,23}$ y considerando que el progreso de dichas creencias se logra, entre otros, dada las experiencias formativas ${ }^{10,16,34}$, las carreras de Medicina deberían poner particular atención en seleccionar e implementar enfoques pedagógicos, 
metodologías y entornos académicos que profilácticamente eviten el retroceso epistemológico. En particular en los últimos años del pre-grado, dado que los profesionales del siglo XXI deben ser autónomos y autoregulados, deben aprender colaborativamente, deben pensar críticamente, deben tomar decisiones basadas en evidencia y deben alcanzar un desempeño profesional flexible.

Para seguir avanzando en este campo, sería un aporte diseñar y evaluar intervenciones tendientes a mantener las creencias epistemológicas sofisticadas durante todo el trayecto formativo de la carrera de Medicina.

Agradecimientos: Los autores agradecen a todos los estudiantes que voluntariamente aceptaron participar en el estudio, a los Directores de carreras que autorizaron la ejecución de él y a los docentes que permitieron aplicar el cuestionario de creencias epistemológicas al inicio de sus clases.

\section{Referencias}

1. Hofer BK. Personal epistemology as a psychological and educational construct: An introduction. In B. K. Hofer, \& P. P. Pintrich (Eds), Personal epistemology: the psychology of beliefs about knowledge and knowing. Nueva Jersey, United States: Lawrence Erlbaum Associates, Inc.; 2002. p. 3-14.

2. Schommer-Aikins $M$, Beuchat-Reichardt M, Hernández-Pina F. Creencias epistemológicas y de aprendizaje en la formación inicial de profesores. An Psicol 2012; 28 (2): 465-74.

3. Perry WG, Sprinthall NA, Wideman JM. Patterns of development in thought and values of students in a liberal arts college: a validation of a scheme. Final Report 1968. Cambridge, MA: Bureau of Study Counsel, Harvard University. (ERIC Document Reproduction Services No ED 024315).

4. Kitchener KS, King PM. Reflective judgment: concepts of justification and their relationship to age and education. J Appl Dev Psychol 1981; 2 (2): 89-116.

5. Belenky MF. Women's ways of knowing: the development of self, voice and mind. Nueva York, United States: Basic Books; 1986.

6. Baxter M. Knowing and reasoning in college: gender-related patterns in students' intellectual development. San Francisco, United States: Jossey Bass Publishers; 1992.

7. Ariza RP, García AR, Pozo RM. Conocimiento profesio- nal y epistemología de los profesores I: Teoría, métodos e instrumentos. Rev Investig Exp Didácticas 1997; 15 (2): 155-71.

8. Pecharromán I, Pozo JI. Cómo sé que es verdad?: epistemologías intuitivas de los estudiantes sobre el conocimiento científico. Investig Em Ensino Cienc 2006; 11 (2): 153-87.

9. Schommer-Aikins M. Explaining the epistemological belief system: introducing the embedded systemic model and coordinated research approach. Educ Psychol 2004; 39 (1): 19-29.

10. Hofer BK, Pintrich PR. The development of epistemological theories: beliefs about knowledge and knowing and their relation to learning. Rev Educ Res 1997; 67 (1): 88-140.

11. Schommer M. Effects of beliefs about the nature of knowledge on comprehension. J Educ Psychol 1990; 82 (2): 498-504.

12. Tolhurst $\mathrm{D}$. The influence of learning environments on students' epistemological beliefs and learning outcomes. Teach High Educ 2007; 12 (2): 219-33.

13. Garner R, Alexander PA. Beliefs about text and instruction with text. Hillsdale, United States: Lawrence Erlbaum Associates, Inc; 1994.

14. Juanas ÁD, Beltrán J. Epistemological beliefs of students of pedagogy and sciences of the education. J Psycho Didactics 2011; 17 (1): 1-15.

15. García MR, Sebastián C. Creencias epistemológicas de estudiantes de pedagogía en educación parvularia, básica y media: ¿Diferencias en la formación inicial docente? Psykhe 2011; 20 (1): 29-43.

16. Sánchez M del R. Creencias epistemológicas de estudiantes de medicina. AVFT 2009; 28 (1): 31-5.

17. Duell OK, Schommer-Aikins M. Measures of people's beliefs about knowledge and learning. Educ Psychol Rev 2001; 13 (4): 419-49.

18. Ryan MP. Monitoring text comprehension: individual differences in epistemological standards. J Educ Psychol 1984; 76 (2): 248-58.

19. Kardash CM, Howell KL. Effects of epistemological beliefs and topic-specific beliefs on undergraduates' cognitive and strategic processing of dual-positional text. J Educ Psychol 2000; 92 (3): 524-35.

20. Schommer M. Epistemological development and academic performance among secondary students. J Educ Psychol 1993; 85 (3): 406-11.

21. Hammer D, Elby A. Epistemological resources. In B. Fishman \& S. O’Connor-Divelbiss (Eds.), Personal Epistemology: The Psychology of Beliefs about Knowledge and Knowing. Nueva Jersey, United States: Lawrence Erlbaum Associates, Inc.; 2000. p. 169-90. 
22. Kember D. Beliefs about knowledge and the process of teaching and learning as a factor in adjusting to study in higher education. Stud High Educ 2001; 26 (2): 20521.

23. Cano F. Epistemological beliefs and approaches to learning: their change through secondary school and their influence on academic performance. Br J Educ Psychol 2005; 75 (2): 203-21.

24. Lazcano X, Santa Cruz J, Conget P. Teorías implícitas sobre la enseñanza y su asociación con las prácticas pedagógicas de los docentes de la carrera de Medicina. Rev Educ Cienc Salud 2013; 10 (1): 47-52.

25. Di Matteo M. Creencias epistemológicas acerca de la naturaleza del conocimiento en estudiantes de ciencias de la educación y profesorados universitarios. IV Jornadas de Jóvenes Investigadores. Instituto de Investigaciones Gino Germani, Facultad de Ciencias Sociales, Universidad de Buenos Aires, Buenos Aires. 2002. Disponible en http://www.aacademica.org/000-024/178 [Consultado el 9 de noviembre de 2016].

26. McEwen MK. Review of the book Knowing and reasoning in college: gender-related patterns in students' intellectual development. NASPA J 1994; 31: 153-7.

27. King PM, Kitchener KS. Reflective judgment: theory and research on the development of epistemic assumptions through adulthood. Educ Psychol 2004; 39 (1): 5-18.

28. Chan KW. Hong Kong teacher education students' epistemological beliefs and approaches to learning. Res Educ 2003; 69 (1): 36-50.

29. Hofer BK. Dimensionality and disciplinary differences in personal epistemology. Contemp Educ Psychol 2000; 25 (4): 378-405.

30. Cano F, Cardelle-Elawar M. An integrated analysis of secondary school students' conceptions and beliefs about learning. Eur J Psychol Educ 2004; 19 (2): 167-87.

31. Rodríguez L. Análisis de las creencias epistemológicas, concepciones y enfoques de aprendizaje de los futuros profesores [tesis doctoral en internet]. Granada: Universidad de Granada; 2005. Disponible en: http://hera.ugr. es/tesisugr/15480112.pdf.

32. Kuhn D. The skills of argument. Cambridge, England. Cambridge University Press; 1991. p. 336.

33. Guerra Zamora P. Análisis del cambio en las creencias sobre el conocimiento, el aprendizaje y la enseñanza en estudiantes de pedagogía en el contexto de su formación inicial [tesis doctoral en internet]. Santiago: Pontificia Universidad Católica de Chile; 2013. Disponible en: http://dspace2.conicyt.cl/bitstream/ handle/10533/91470/GUERRA_PAULA_2617D.pdf?sequence $=1$

34. Chan KW, Elliott RG. Exploratory study of epistemological beliefs of Hong Kong teacher education students: resolving conceptual and empirical issues. Asia-Pac J Teach Educ 2000; 28 (3): 225-34. 